\title{
Erlotinib Hydrochloride
}

National Cancer Institute

\section{Source}

National Cancer Institute. Erlotinib Hydrochloride. NCI Thesaurus. Code C2693.

The hydrochloride salt of a quinazoline derivative with antineoplastic properties.

Competing with adenosine triphosphate, erlotinib reversibly binds to the intracellular catalytic domain of epidermal growth factor receptor (EGFR) tyrosine kinase, thereby reversibly inhibiting EGFR phosphorylation and blocking the signal transduction events and tumorigenic effects associated with EGFR activation. 